\title{
Spatial routing network building for pedestrian panoramic survey points when monitoring urban areas
}

\author{
Ignat N. Penshin ${ }^{1,}$, Larisa G. Evstratova ${ }^{1}$ \\ ${ }^{1}$ State University of Land Use Planning, 105064, 15 Kazakova str., Moscow, Russian Federation
}

\begin{abstract}
The relevance of the development of new solutions for building a spatial routing network for points of pedestrian panoramic shooting when monitoring urban infrastructure is considered. A computationally efficient automated algorithm has been developed and implemented in software, which provides the ability to move the user in the forward and backward direction between any pair of incident points of a panoramic survey using a custom geoportal. The proposed approach takes into account changes in the urban environment (transport networks, capital structures), thus, they are not an obstacle to the operation of the created spatial routing network. It remains possible to rebuild the network at any required scale (shooting tracks, groups of tracks, urban areas, administrative districts, the whole city).
\end{abstract}

\section{Introduction}

Today, there are various web services for viewing street panoramas, in which images obtained by means of automobile panoramic shooting are available. However, this does not provide a complete overview of urban areas [1]. This disadvantage can be eliminated by pedestrian panoramic photography, since it provides the maximum possible territorial coverage due to the high mobility of the operator performing the shooting, i.e. positioning independent of the road network. In this regard, the automation of spatial routing within courtyard areas for points of pedestrian panoramic survey becomes relevant.

The effectiveness of urban resource management depends on the complete and timely receipt of information about the objects of urban infrastructure, which is achieved through monitoring [2]. A georeferenced and spatial-oriented panoramic image allows to get up-todate information about the city space. Interpretation of the obtained data array during panoramic shooting within the framework of geographic information system allows to conduct an inventory and subsequent analysis of the compliance of certain territories using with the intended purpose [3, 4].

\footnotetext{
${ }^{1}$ Corresponding author: ignat.penshin51@gmail.com
} 


\section{Navigation network construction algorithm for pedestrian panoramic survey points}

The functional block of an intelligent GIS agent contains an algorithm that generates a set of rational solutions when creating spatial routing inside courtyard areas without using information about the road network [5].

The algorithm takes initial data as input and returns a set of links between point objects. Figure 1 shows a block diagram of the sequence of actions of the algorithm for spatial routing network construction. Next, we will consider the main factors affecting the complexity of the algorithm.

\section{NAVIGATION NETWORK CONSTRUCTION ALGORITHM}

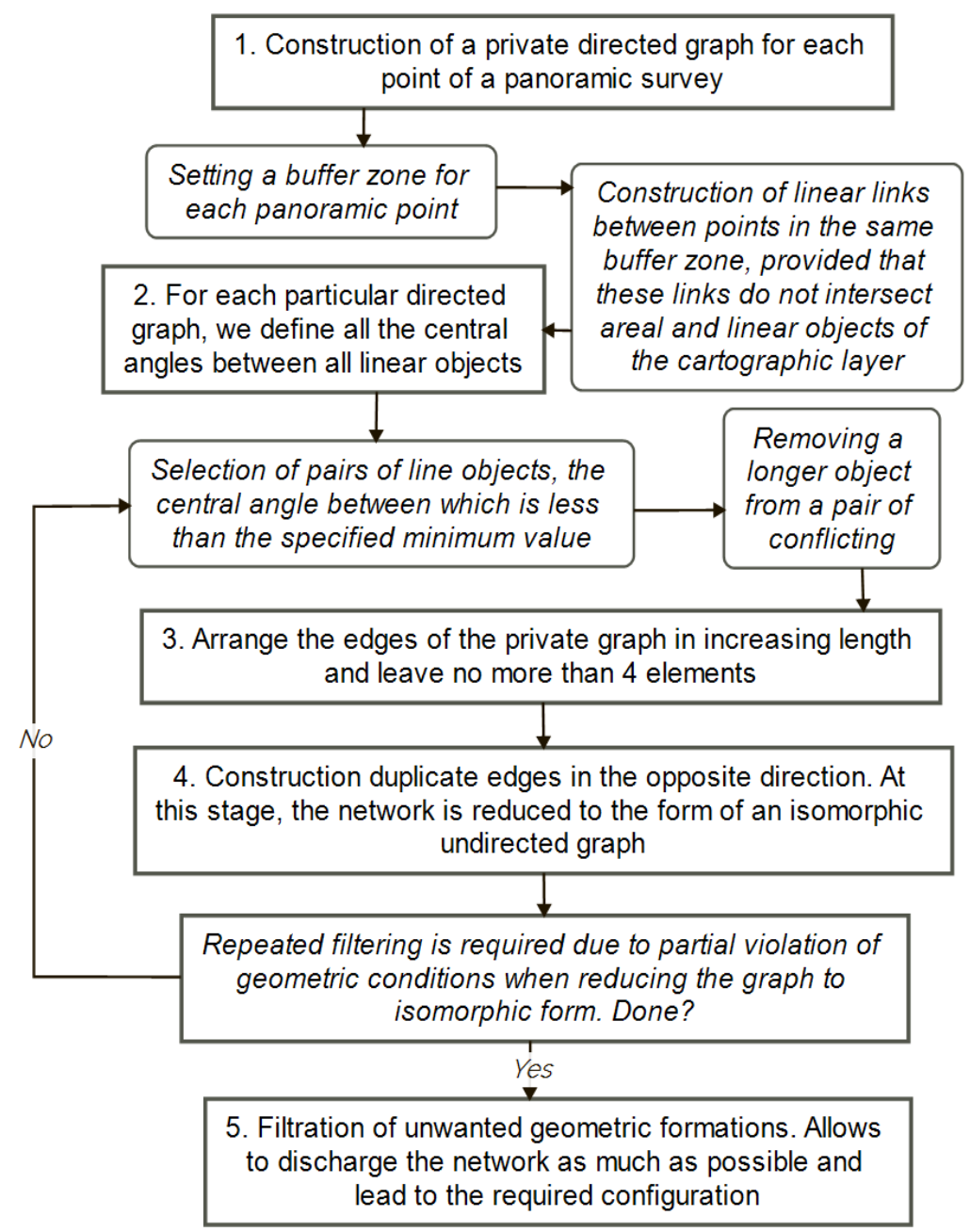

Fig. 1. Block diagram of the spatial routing network construction algorithm 


\section{Analysis of the decision rules complexity}

The greatest contribution to the complexity of the algorithm is made by the execution of 1 decision rule. The choice of the optimal parameters for its execution significantly reduces the amount of computation. Let us consider in more detail the order of execution of 1 decision rule, and also define the input sets of objects:

During the execution of decision rule 1, a buffer zone with a radius equal to the variable of maximum distance between points (buff_radius = dist_max) is created for each panoramic point (Panoramas table). Neighborhood points (Panoramas table), intersected by the buffer zone of this point, are used to create an array of linear objects with the beginning at this point - thus we get a private directed graph for this point [5]. Next, line objects crossing linear (Highways table) or area objects (Buildings table) that are in the buffer of this point are deleted. The described sequence of decision rule 1 actions is performed for each point of the Panoramas table.

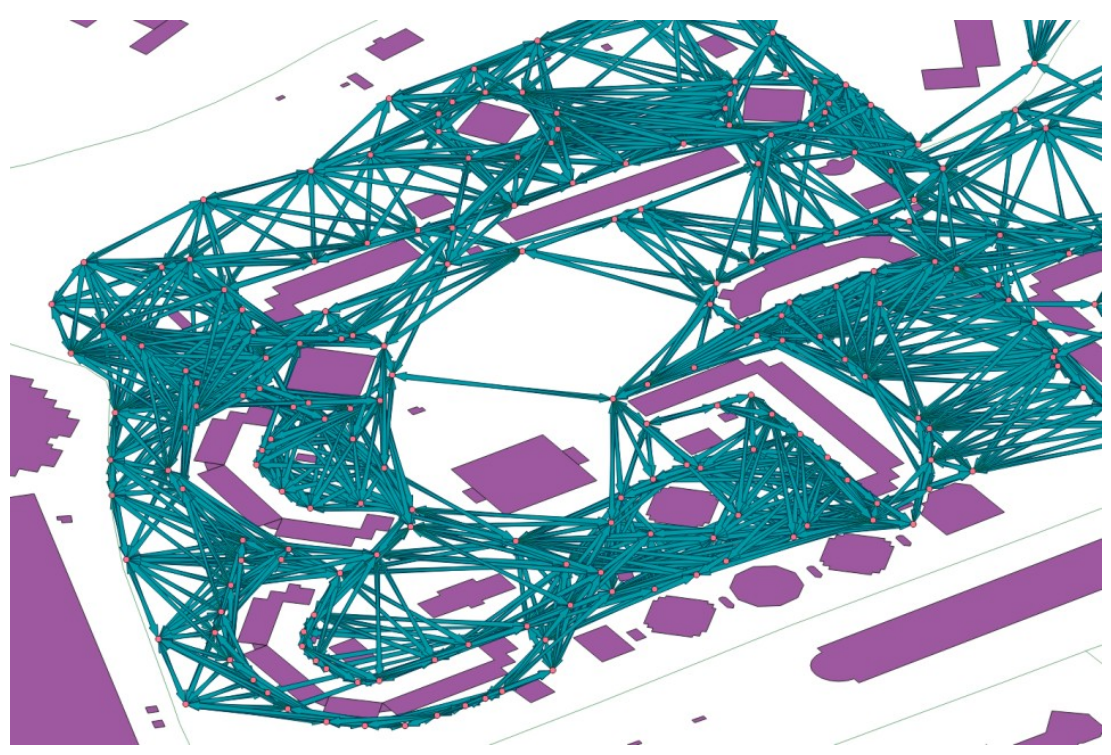

Fig. 2. Execution of the 1 decision rule of the spatial routing network construction algorithm

Let us define the set of Panoramas photographing points as $\mathrm{V}$, the set of areal objects Buildings - B, the set of line objects Highways - H, the set of connecting edges Edges - E. If an object of any of the sets $\mathrm{V}, \mathrm{B}, \mathrm{H}$ is in the intersection with the buffer of the given initial point $v_{j}$, then this object is considered when forming a private graph for this initial point and falls into the subset $\Gamma_{v_{j}}$. We will use the mathematical apparatus of graph theory and define subsets of a given initial point $v_{j}(1-3)$ :

$$
\begin{aligned}
& \Gamma_{v_{j}}(u)=\left\{\forall u_{i} \in u \in V \mid \max s\left(u_{i}, v_{j}\right) \leq \operatorname{dist}_{\text {max }}\right\} \\
& \Gamma_{v_{j}}(b)=\left\{\forall b_{i} \in b \in B \mid \max s\left(b_{i}, v_{j}\right) \leq \operatorname{dist}_{\text {max }}\right\} \\
& \Gamma_{v_{j}}(h)=\left\{\forall h_{i} \in h \in H \mid \max s\left(h_{i}, v_{j}\right) \leq \operatorname{dist}_{\max }\right\}
\end{aligned}
$$


To determine the above subsets, it is necessary to perform a sequential enumeration of all elements of the sets $\mathrm{V}, \mathrm{B}, \mathrm{H}$ relative to each photographing point as the initial one. The number of elements of this set is directly proportional to the number of operations performed and is determined by its capacity. Then the complexity of performing the first action $M_{1.1}$ to determine the subsets of each point is determined by the formula (4):

$$
M_{1.1}=(|V|+|B|+|H|) *|V|
$$

After defining the subsets $\Gamma_{v_{j}}(u), \Gamma_{v_{j}}(b), \Gamma_{v_{j}}(h)$, the subset of connecting edges $E_{j}$, implemented as an array of linear objects, is determined. This subset is described by formula (5):

$$
E_{j}=\left\{\forall u_{i} \in \Gamma_{v_{j}}(u) \hookrightarrow\left(v_{j}, u_{i}\right)\right\}
$$

Satisfaction of the condition of absence of intersection of connecting edges $E_{j}$ and objects of subsets $\Gamma_{v_{j}}(b), \Gamma_{v_{j}}(h)$ is defined by the subset of edges $E_{j}^{\prime} \in E_{j}$. The subset $E_{j}^{\prime}$ corresponds to a logic judgment of the form: the set of edges $E_{j}$ that do not belong to the subset of areal objects $\Gamma_{v_{j}}(b)$ AND the set of edges $E_{j}$ that do not belong to the subset of linear objects $\Gamma_{v_{j}}(b)$. The logical notation $E_{j}^{\prime}$ will take the form (6):

$$
E_{j}^{\prime}=E_{j} \backslash\left(\forall b_{i} \in \Gamma_{v_{j}}(b)\right) \wedge E_{j} \backslash\left(\forall h_{i} \in \Gamma_{v_{j}}(h)\right)
$$

To implement the condition of the absence of intersections and the formation of the subset $E_{j}^{\prime}$, it is necessary to perform a sequential enumeration of the area objects Buildings from the subset $\Gamma_{v_{j}}(b)$ and linear objects Highways from the subset $\Gamma_{v_{j}}(h)$ for connecting edges from the set $E_{j}$. The complexity of the second action $M_{1.2}$ is determined by the formula (7):

$$
M_{1.2}=\sum_{j=1}^{\left|E_{j}\right|}\left(\left|\Gamma_{v_{j}}(b)\right|+\left|\Gamma_{v_{j}}(h)\right|\right)
$$

The complexity of the sequential performance of actions in the first decision rule can be estimated by the formula (8):

$$
M_{1}=\left(|V|+|B|+|H|+\sum_{j=1}^{\left|E_{j}\right|}\left(\left|\Gamma_{v_{j}}(b)\right|+\left|\Gamma_{v_{j}}(h)\right|\right)\right) *|V|
$$

From formula (8) it follows that the number of operations performed is directly proportional to the size of the processed area, namely, the number of survey points, construction objects and roads included in the area. If we assume that the difference in resource intensity and execution time of computational operations is negligible, then we can compare the number of operations for processing input data arrays that differ in size. Further actions will make it possible to determine the optimal size of a unit processing area based on an a priori estimate of the complexity of the decision rule 1 execution by an intelligent agent $[6,7]$.

According to the averaged performance indicators of the field group of operators of pedestrian panoramic survey, as well as separate averaged representations of geodata, we will use the following constant values of the quantities involved in determining the complexity of the first decision rule (Table 1). 
Table 1. Constants in determining the complexity of decision rule 1

\begin{tabular}{|c|c|}
\hline Value & Quantity \\
\hline $\begin{array}{l}\overline{\left|E_{J}\right|} \text { - the average number of Panoramas vertices falling into } \\
\text { the buffer zone of this initial vertex }\end{array}$ & $\sim 8$ \\
\hline $\begin{array}{l}\left|\Gamma_{v_{J}}(b)\right| \text { - the average number of areal Buildings objects that fall } \\
\text { into the buffer zone of this initial vertex }\end{array}$ & $\sim 3$ \\
\hline $\begin{array}{l}\left|\Gamma_{v_{J}}(h)\right| \\
\text { within the the average number of Highways line features that fall } \\
\text { of this initial vertex }\end{array}$ & $\sim 2$ \\
\hline
\end{tabular}

Let's assume that, on average, the territory of one block contains: 10 areal Buildings, 4 adjacent highways, 200 Panoramas panoramic points. The calculation of the complexity of fulfilling 1 decision rule according to formula (8) for territories that are multiples of the size of one quarter is presented in Table 2.

Table 2. Decision rule complexity 1 for input Datasets

\begin{tabular}{|c|l|l|l|l|l|l|}
\hline Sets & Dataset 1 & Dataset 2 & Dataset 3 & Dataset 4 & Dataset 5 & Dataset 6 \\
\hline$|V|$ & 5000 & 2500 & 1000 & 500 & 250 & 100 \\
\hline$|B|$ & 250 & 125 & 50 & 25 & 12 & 5 \\
\hline$|H|$ & 100 & 50 & 20 & 10 & 5 & 2 \\
\hline Complexity (number of operations) \\
\hline$M_{1}$ & 26950000 & 6787500 & 1110000 & 287500 & 76750 & 14700 \\
\hline
\end{tabular}

According to the values of $M_{1}$ for different sets of input data, the most optimal in terms of time and productivity is the yard processing of the territory when the decision rule 1 is fulfilled. The optimal sets correspond to Set 5 and Set 6 of Table 2.

\section{Conclusion}

Experiments on the performance of the proposed spatial routing algorithm for panoramic survey points show that the proposed approach generates a set of rational solutions when creating a spatial routing network inside courtyard territories without using information about the road network and the most optimal in terms of time and productivity is quarterly processing of the territory. As a result, the geoportal, which aggregates and provides the user with georeferenced and spatial-oriented panoramic images, makes it possible to solve the following applied problems: analytics in the field of urban planning without the need for physical presence in the study area; inventory of objects of engineering, transport, social, industrial, residential infrastructure, development of the recreational potential of territories, etc.

\section{References}

1. Nam, T., \& Pardo, Conceptualizing smart city with dimensions of technology, people, and institutions. In Proceedings of the 12th annual international digital government research conference: digital government innovation in challenging times, 282 (2011)

2. Signore R., Stegman M. O., Creamer J. The ODBC solution: Open database connectivity in distributed environments. - McGraw-Hill, Inc., 1995

3. Fotheringham, S., Rogerson, P., Spatial analysis and GIS (2014) 
4. Hand, D. J., Data Mining, Encyclopedia of Environmetrics, 2 (2006)

5. Penshin I. N., Evstratova L. G. Navigation network construction method for survey points using cartographic urban environment information //E3S Web of Conferences, . 149, 02001, (2020)

6. Xiaoping D. P. Y. C. R., Jiliang G. Algorithm of Spatial Select Query in GIS Based on the Improved Quadtree with the Case of ESRI SHAPE File [J]//Computer Engineering and Applications, 13 (2003)

7. Piórkowski A. et al. Mysql spatial and postgis-implementations of spatial data standards //Electronic journal of Polish agricultural universities, 14, 1-8 (2011). 